\title{
CORRECTION
}

\section{More than naming of parts}

Nature Plants 1, 15086 (2015); published online 2 June 2015; corrected 4 June 2015

In the version of this Editorial originally published, the reference to the Comment should have cited A. J. Miller et al. This has been corrected. 